\title{
Correction to: Higher-order Operators on Networks: Hyperbolic and Parabolic Theory
}

Federica Gregorio and Delio Mugnolo@

\section{Correction to: Integr. Equ. Oper. Theory (2020) 92:50 https://doi.org/10.1007/s00020-020-02610-8}

In the original version of this article, the $\$$ symbols were misinterpreted due to a production error as part of the article text and placed in the article as it is. The symbols were a part of the corrections to the roman numerals in several places. This has now been corrected.

The original article has also been updated.

Publisher's Note Springer Nature remains neutral with regard to jurisdictional claims in published maps and institutional affiliations.

Federica Gregorio

Dipartimento di Matematica

Universitá degli Studi di Salerno

Via Giovanni Paolo II, 132

84084 Fisciano SA

Italy

e-mail: fgregorio@unisa.it

Delio Mugnolo $(\varangle)$

Lehrgebiet Analysis, Fakultät Mathematik und Informatik

Fern Universität in Hagen

58084 Hagen

Germany

e-mail: delio.mugnolo@fernuni-hagen.de

The original article can be found online at https://doi.org/10.1007/s00020-020-02610-8. 